\title{
Organische Kohlenstoffvorräte von Bodentypen in den Hauptnaturräumen Schleswig-Holsteins (Norddeutschland)
}

\section{Organic carbon stocks of soil types in the geological regions of Schleswig-Holstein (Northern Germany)}

\author{
Anneka Mordhorst ${ }^{1 *}$, Heiner Fleige ${ }^{1}$, Iris Zimmermann ${ }^{1}$, Bernd Burbaum², Marek Filipinski², \\ Eckhard Cordsen ${ }^{2}$, Rainer Horn ${ }^{1}$
}

\footnotetext{
${ }^{1}$ Institut für Pflanzenernährung und Bodenkunde, Christian-Albrechts-Universität Kiel, Hermann Rodewaldstraße 2, 24118 Kiel, Deutschland

${ }^{2}$ Landesamt für Landwirtschaft, Umwelt und ländliche Räume Schleswig-Holstein (LLUR), Hamburger Chaussee 25, 24220 Flintbek, Deutschland

* Korrespondierende Autorin: a.mordhorst@soils.uni-kiel.de
}

Einreichung: 26. April 2018, überarbeitete Einreichung: 2. Juli 2018, Annahme: 5. Juli 2018

\section{Zusammenfassung}

Die Verbesserung der Humusspeicherung in Böden im Hinblick auf eine nachhaltige Landbewirtschaftung erfordert genaue Kenntnisse über den organischen Kohlenstoff $\left(\mathrm{C}_{\text {org }}\right)$-Vorrat in Böden, wobei auch die Wechselwirkungen zwischen Bodentyp, geologischem Ausgangsgestein sowie Landnutzung berücksichtigt werden müssen. Auf der Grundlage von insgesamt 925 Bodenprofilen in den vier Hauptnaturräumen (Östliches Hügelland, Vorgeest, Hohe Geest und Marsch) Schleswig-Holsteins (Norddeutschland) wurde der $\mathrm{C}_{\text {org }}$-Vorrat bis in eine Tiefe von $90 \mathrm{~cm}$ quantifiziert. Die horizontspezifischen Parameter (u. a. auch Trockenrohdichte und Korngrößenverteilung) wurden im Rahmen der seit ca. 1970 durchgeführten bodenkundlichen Leitprofilaufnahmen vom Landesamt für Landwirtschaft, Umwelt und ländliche Räume Schleswig-Holstein (LLUR) und deren Vorgängerbehörden erhoben. Die vier Hauptnaturräume enthalten unterschiedliche $C_{o r g}$-Vorräte in den Bodentiefen 0-30, 30-60 und 60-90 cm. Insgesamt werden in Schleswig-Holstein mindestens $244 \mathrm{Mt} \mathrm{C}_{\text {org }}(0-90 \mathrm{~cm}$ Tiefe) gespeichert, wobei je nach Landnutzung, Bodentyp und geologischem Ausgangsgestein deutliche Unterschiede auftreten. Im Oberboden variieren die $\mathrm{C}_{\text {org }}$-Vorräte von ca. 60 t/ha (Ackerböden der Marsch/des Östlichen Hügellands) bis ca. 130 t/ha (Grünlandböden der Geest). Im Unterboden verfügen neben den Mooren die humusreichen Marschböden, die Kolluvisole (Alt- und Jungmoränengebiet) und Gley-Podsole (Hohe Geest und Vorgeest) über die höchsten $\mathrm{C}_{\text {org }}$-Vorräte bis $90 \mathrm{~cm}$ Tiefe. Das Speicher- und Verlustpotenzial von Humusmengen durch einen Landnutzungswechsel ist daher auch bodentypspezifisch zu bewerten.

Schlagworte: Organischer Kohlenstoffvorrat $\left(\mathrm{C}_{\text {org }}\right)$, Landnutzung, geologisches Ausgangsgestein, Mineralisation, Klimawandel

\section{Summary}

The improvement of carbon sequestration in soils for a more sustainable land use management requires knowledge about soil organic carbon (SOC) stocks as well as the interactions between soil type, parent material and land use. In Northern Germany (federal state: Schleswig-Holstein) about 925 soil profiles in the four geological regions (Weichselian glacial region, Lower "Geest", Higher "Geest" and the marshland) were sampled down to a depth of at least $90 \mathrm{~cm}$. Horizon-specific parameters like bulk density and grain size distribution were analyzed among others during several soil inventory programs by the (State) Agency for Agriculture, Environment and Rural Areas of the German Federal State Schleswig-Holstein (LLUR) as well as their predecessor agencies since 1970. The four geological regions possess different SOC stocks for the soil depths 0-30, 30-60, 60-90 cm. In total, at least $244 \mathrm{Mt}$ SOC is stored down to a depth of $90 \mathrm{~cm}$ while the amount differs depending on the land use type, soil type and parent material. In the topsoil $(0-30 \mathrm{~cm}$ ), SOC varied from $60 \mathrm{t} / \mathrm{ha}$ (arable soils of Weichselian glacial area and marshland) up to $130 \mathrm{t} / \mathrm{ha}$ (grassland soils in the Geest areas). Besides the Histosols, the highest SOC stocks in the subsoil (30-90 cm depth) were attributed to the marshland soils, Anthrosols ("Colluvic", Weichselian glacial region and Higher "Geest") and Gleyic Podzols (Lower and Higher "Geest"). Evaluating the potential of SOC storage and loss by land use changes, soil type-specific SOC stocks should be taken into account.

Keywords: Soil organic carbon (SOC) stocks, land use, parent material, mineralization, climate change 


\section{Einleitung}

Die Humus-/Kohlenstoffspeicherung und die Änderung der organischen Kohlenstoffgehalte $\left(\mathrm{C}_{\text {org }}\right)$ in Böden werden gegenwärtig sehr intensiv diskutiert. Die von einer Vielzahl von Staaten ratifizierte „4 pro Tausend Initiative (quatre per mille)“ der französischen Regierung anlässlich der Vertragsstaatenkonferenz (Conference of the Parties, COP) in Paris führt derzeit zu einer weltweit steigenden Aufmerksamkeit hinsichtlich der Erreichung der Klimaziele, welche in den „Sustainable Development Goals (SDG)" präzisiert worden sind (IPCC, 2006; 4p1000, 2017; Conrad et al., 2017; van Groenigen et al., 2017; Zomer et al., 2017). Neben der Erfassung der aktuellen Vorräte gilt es auch die Auswirkungen der Bodenbewirtschaftung für die Speicherung und damit die Entlastung der Atmosphäre von $\mathrm{CO}_{2}$-Emissionen im In- und Ausland zu quantifizieren, um darüber eine bodenschonende und standortangepasste Landbewirtschaftung im Hinblick auf die Einhaltung bzw. Erreichung der Klimaziele zu definieren (Balesdent und Arrouays, 1999; Cosentino et al., 2006; Wiesmeier et al., 2012; Chenu et al., 2018). Diese Quantifizierung geschieht auch vor dem Hintergrund einer durch Humus verbesserten Bodenstruktur, günstigeren Wasser-, Nährstoff- und Gaszusammensetzung, eines gleichmäßigen Wärmehaushalts sowie einer höheren mikrobiellen Aktivität im Boden, die gleichzeitig sogar zum Aufbau stabiler C-Verbindungen im Boden beitragen kann (Liang et al., 2017). Darüber hinaus erhöhen die bei der Mineralisation freigesetzten organischen Säuren über die Bildung organo-mineralischer Verbindungen die mechanische Stabilität, wobei je nach Zusammensetzung der organischen Säuren durchaus große Unterschiede hinsichtlich der mechanischen Stabilisierung auftreten können (Blume et al., 2010; Hartge und Horn, 2014).

Es muss allerdings berücksichtigt werden, dass bei kontinuierlicher Bodenbearbeitung auf Ackerböden nicht von einer gleichmäßig steigenden Humusspeicherung ausgegangen werden kann, da im Zuge der Mineralisierung der organischen Pflanzenreste durch die Mikroorganismen wiederum Nährstoffe für die nachwachsenden Pflanzen im Boden zur Verfügung gestellt werden. Von daher ist nur mit einer begrenzten zusätzlichen Speicherung von $\mathrm{C}_{\text {org }}$ im Boden zu rechnen. In diesem Kontext berechneten Lugato et al. (2014), dass ein Umstieg auf alternative Bodenbewirtschaftungsformen (z. B. reduzierte Bodenbearbeitung, Zwischenfruchtanbau, Futtergräser/Leguminosen als Fruchtfolgeglieder, kein vollständiger Abtransport von Ernterückständen) für mindestens $12-28 \%$ der Ackerböden Europas notwendig ist, um die gesetzten Klimaziele 2020 hinsichtlich der $\mathrm{CO}_{2}$-Einsparung zu erreichen. Abgesehen von der Bodenbewirtschaftung ist die Bodentextur (Tongehalt) und damit auf jungen Landoberflächen wie dem norddeutschen Tiefland das geologische Ausgangsmaterial für die C-Speicherkapazität und das langfristige C-Speicherungspotenzial der Böden von Bedeutung (Six et al., 2002; Chen et al., 2018). Ein Überblick über die weltweiten Humusvorräte in den obersten $30 \mathrm{~cm}$ lässt sich aus der anlässlich der FAO-Tagung in Rom 2017 publizierten $\mathrm{C}_{\text {org }}$-Vorratskarte ableiten (FAO, 2017). Hierbei muss berücksichtigt werden, dass der Kohlenstoffvorrat in den obersten $30 \mathrm{~cm}$ unter Geländeoberfläche (GOF) aufgrund der Bodenbearbeitung besonders intensiven Veränderungen unterworfen ist. Nach der gegenwärtigen Berechnung befinden sich weltweit $677 \mathrm{Pg}\left(=677^{*} 10^{9} \mathrm{t}\right) \mathrm{C}_{\text {org }}$ in den obersten $30 \mathrm{~cm}$ mit allerdings deutlichen Unsicherheiten. Bezogen auf $1 \mathrm{~m}$ Tiefe variieren die berechneten $\mathrm{C}_{\text {org }}$-Vorräte weltweit zwischen 1400-1600 Pg (Batjes, 2016). Gemessene $C_{\text {org }}$-Vorräte bis $1 \mathrm{~m}$ Bodentiefe sind allerdings relativ wenig publiziert, obwohl gerade diese Vorräte für eine langfristige Humusspeicherung von Bedeutung sind, da die organische Bodensubstanz (Humus) im Unterboden für Mikroorganismen schlechter zugänglich (z. B. C-Okklusion in Aggregaten) und damit stärker vor mikrobiellem Abbau (Mineralisation) geschützt ist als im Oberboden (Rumpel und KögelKnabner, 2011; Chen et al., 2018).

\section{Material und Methoden}

\subsection{Untersuchungsgebiet}

Um einen Überblick über die in Schleswig-Holstein gespeicherten Humus-/ $\mathrm{C}_{\text {org }}$-Vorräte bis in $90 \mathrm{~cm}$ Tiefe $\mathrm{zu}$ bekommen, wurden die vom Landesamt für Landwirtschaft, Umwelt und ländliche Räume Schleswig-Holstein (LLUR) und deren Vorgängerbehörden in den zurückliegenden Jahrzehnten (seit ca. 1970) erhobenen Profildaten (LLUR-Datenbank) ausgewertet. Diese umfassen Datensätze von 925 Bodenprofilen aus den vier Hauptnaturräumen Schleswig-Holsteins: Östliches Hügelland, Vorgeest, Hohe Geest und Marsch. Unterschieden wurde hierbei zwischen der Landnutzung zum Zeitpunkt der Beprobung unterteilt in Ackerland, Grünland und Wald.

Im Östlichen Hügelland (Jungmoränenlandschaft, weichselkaltzeitlich geprägt) dominieren Braunerden, (Pseudog- 
ley-)Parabraunerden, Pseudogleye, Kolluvisole und Niedermoore, in der Vorgeest (Sanderebene) vor allem sandige Braunerden, (Gley-)Podsole und Gleye neben Nieder- und Hochmooren. Die Hohe Geest (Altmoränenlandschaft, durch die Saale-Kaltzeit geprägt) wird von lehmig-sandigen und sandigen, carbonatfreien Braunerden, Podsolen, Pseudogleyen und Gleyen neben sauren Niedermooren, Kolluvisolen und Plaggeneschen dominiert. Das Gebiet der Marschen umfasst Roh- (= Salz-), Kalk-, Klei-, Knick-, Dwog- und Organomarschen sowie Nieder- und Hochmoore. Die regionale Verteilung der repräsentativen Bodentypen (> 3 \% Flächenanteil im Hauptnaturraum) und die jeweiligen Flächenanteile sind auf Grundlage der Bodenübersichtskarte 1:250.000 (BÜK 250) (LLUR, 2016) in Tabelle 1 zusammengestellt. Das Östliche Hügelland umfasst eine Fläche von $6740 \mathrm{~km}^{2}$, die Hohe Geest von $3766 \mathrm{~km}^{2}$, die Vorgeest von $2010 \mathrm{~km}^{2}$ und die Marsch von $2854 \mathrm{~km}^{2}$.

\subsection{Labor- und Datenanalyse}

Folgende chemische und physikalische Kenngrößen aus der LLUR-Datenbank wurden in die Auswertung einbezogen: Korngrößenverteilung (prozentuale Anteile der Sand-, Schluff- und Tonfraktion), Humusgehalt bzw. organischer Kohlenstoffgehalt $\left(\mathrm{C}_{\text {org }}\right)$ sowie Trockenrohdichte. Methodische Einzelheiten sind Blume et al. (2011) und Hartge und Horn (2014) zu entnehmen. Während die Trockenrohdichte (Trocknung bis zur Gewichtskonstanz bei $105^{\circ} \mathrm{C}$ der Stechzylinderbodenprobe mit definiertem Volumen) über den gesamten Beprobungszeitraum von 1970-2015 nach gleicher Vorgehensweise bestimmt wurde, beruhen die $\mathrm{C}_{\mathrm{org}}$-Werte projektbedingt auf unterschiedlichen Analysemethoden (Elementaranalyse: trockene Veraschung nach DIN ISO 10964; Lichterfelder Methode: nasse Veraschung nach DIN ISO 19684 Teil 2; Bestimmung des Glühverlustes DIN ISO 19684 Teil 3).

Die Analysen wurden an gestörten und ungestörten Bodenproben aus den relevanten Bodenhorizonten (ohne organische Auflagehorizonte) bis in $90 \mathrm{~cm}$ Tiefe durchgeführt.

Die $\mathrm{C}_{\text {org }}$-Vorräte ( $\mathrm{t} / \mathrm{ha}$ ) errechnen sich aus dem $\mathrm{C}_{\text {org }}$-Gehalt (\%) multipliziert mit der Trockenrohdichte $\left(\mathrm{g} / \mathrm{cm}^{3}\right)$ und der jeweiligen Bodenmächtigkeit $(\mathrm{cm})$. Multipliziert mit der jeweiligen Bodenfläche (ha) ergeben sich die absoluten $\mathrm{C}_{\text {org }}$-Vorräte (in Megatonnen $(\mathrm{Mt})=10^{6} \mathrm{t}$, entsprechen 1 Teragramm $(\mathrm{Tg}$, $10^{12} \mathrm{~g}$ ) oder 0,001 Petagramm (Pg, $\left.\left.10^{15} \mathrm{~g}\right)\right)$. Die Ergebnisse wurden für die folgenden Tiefenstufen aufsummiert: 0-30,
30-60, 60-90 sowie 0-90 cm. Da die Beprobung der Bodenleitprofile im Rahmen verschiedener Projekte durchgeführt wurde, variiert die Beprobungstiefe je nach Forschungsschwerpunkt, sodass für die in dieser Arbeit festgelegten Tiefenstufen jeweils unterschiedliche Probenmengen (n) vorliegen.

\section{Ergebnisse}

\subsection{Einfluss des geologischen Ausgangsgesteins und Bodentyps auf den $\mathrm{C}_{\text {org }}$-Vorrat}

Die vier Hauptnaturräume weisen unterschiedliche $\mathrm{C}_{\text {org }}{ }^{-}$ Vorräte auf (Tabelle 1). Insgesamt werden in den obersten $90 \mathrm{~cm}$ in den Böden Schleswig-Holsteins mindestens $244 \mathrm{Mt}\left(244^{*} 10^{6} \mathrm{t}\right.$, entsprechen $\left.0,244 \mathrm{Pg}\right)$ gespeichert, was einer Humusmenge von ca. $420 \mathrm{Mt}$ entspricht. Diese Menge bezieht sich auf die dominierenden Bodentypen in den vier Hauptnaturräumen, die mehr als $90 \%$ der gesamten Bodenoberfläche von $15.369 \mathrm{~km}^{2}$ ausmachen. Die fruchtbaren Böden im Östlichen Hügelland speichern aufgrund der großen Fläche den höchsten Anteil, gefolgt von der Hohen Geest und der Marsch. Der kleinste Hauptnaturraum, die Vorgeest, weist mit $40 \mathrm{Mt}$ absolut gesehen den niedrigsten Wert auf. In Bezug zur Fläche ( $t / h a)$, d. h. hinsichtlich der Kohlenstoffdichte, ergibt sich jedoch ein anderes Bild, wie Tabelle 1 verdeutlicht: So ist der durchschnittliche $\mathrm{C}_{\text {org }}$-Vorrat pro ha für Böden in der Marsch am größten und nimmt in der Reihenfolge Marsch > Vorgeest $>$ Östliches Hügelland > Hohe Geest ab.

Erwartungsgemäß weisen Moore (Nieder- und Hochmoore) die höchsten $\mathrm{C}_{\text {org }}$-Vorräte über die Tiefe auf. Damit sind sie trotz des geringen Flächenanteils (7 \%) für ca. $30 \%$ des gesamten $\mathrm{C}_{\text {org }}$-Vorrats in Schleswig-Holstein (77 Mt/ha bis $90 \mathrm{~cm}$ Tiefe) verantwortlich (Abbildung 1). Ebenfalls hohe $\mathrm{C}_{\text {org }}$-Vorräte sind in den Kolluvisolen zu finden, die $11 \%$ des gesamten $\mathrm{C}_{\text {org }}$-Vorrats in Schleswig-Holstein abdecken. Bei den Kolluvisolen und Mooren handelt es sich um Mindestwerte, da die Mächtigkeiten ( $\mathrm{H}$ - und M-Horizonte) auch mehr als $90 \mathrm{~cm}$ betragen können. Der Kolluvisol, ein für das Östliche Hügelland besonders typischer Bodentyp (16\% Flächenanteil), stellt zusammen mit den dort vorkommenden Niedermooren ( $<4 \%$ Flächenanteil) mehr als die Hälfte des $\mathrm{C}_{\text {org }}$-Speichers dar (Abbildung 1), während die $\mathrm{C}_{\text {org }}$-Vorräte für die übrigen Bodentypen des Östlichen Hügellandes vergleichsweise gering ausfallen. Hier sind z. B. die fruchtbaren Parabraunerden aus Geschiebemergel mit nur $4 \mathrm{Mt} \mathrm{C}_{\text {org }}$ zu nennen (Tabelle 1). 


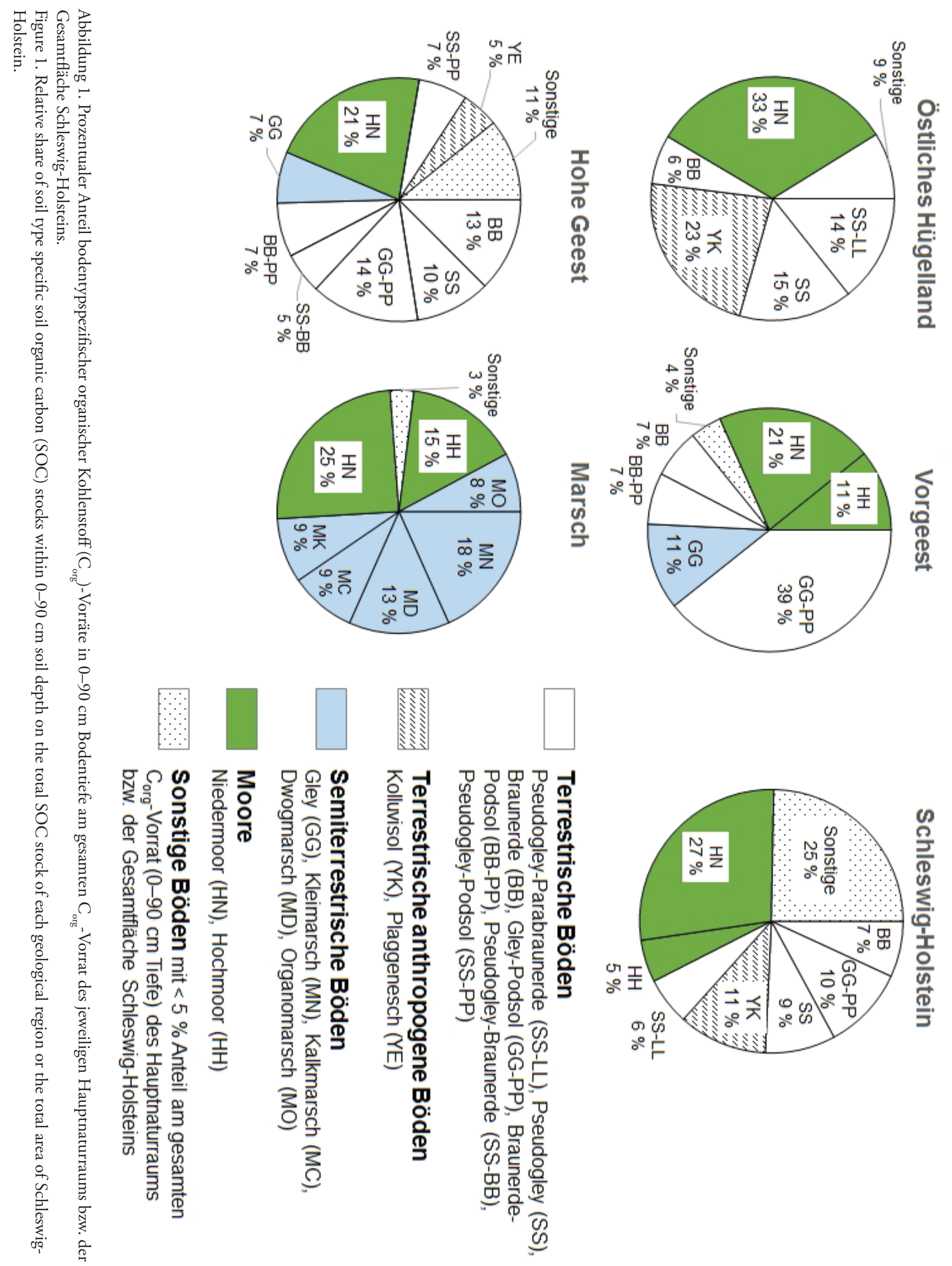


Tabelle 1. Tiefenspezifische organische Kohlenstoffvorräte $\left(\mathrm{C}_{\text {org }}\right)$ in 0-90 cm Bodentiefe von repräsentativen Bodentypen mit einem relativen Flächenanteil von $>3 \%$ in den Hauptnaturräumen Schleswig-Holsteins. $\mathrm{n}=$ Anzahl der Bodenprofile, $\mathrm{x}=$ arithmetischer Mittelwert, $+/-=$ Standardabweichung, Datengrundlage: LLUR.

Table 1. Depth-specific soil organic carbon (SOC) stocks from 0-90 cm depth for the most frequent soil types with a relative area $>3 \%$ in the different geological regions in Schleswig-Holstein. $\mathrm{n}=$ number of soil profiles, $\mathrm{x}=$ arithmetic mean, + $/-=$ standard deviation, data basis: LLUR.

Östliches Hügelland

\begin{tabular}{|c|c|c|c|c|c|c|c|c|c|c|c|c|c|c|c|}
\hline \multirow{3}{*}{ Bodentyp } & \multirow{3}{*}{$\begin{array}{c}\text { Ton }(\mathbf{x}) \\
(\%)\end{array}$} & \multirow{3}{*}{$\begin{array}{c}\text { Ton } \\
(+/-) \\
(\%) \\
\end{array}$} & \multirow{3}{*}{$\begin{array}{c}\text { Rel. } \\
\text { Fläche } \\
(\%) \\
\end{array}$} & \multirow{3}{*}{$\begin{array}{l}\text { Abs. } \\
\text { Fläche } \\
\text { (ha) }\end{array}$} & \multicolumn{3}{|c|}{$0-30 \mathrm{~cm}$} & \multicolumn{3}{|c|}{$30-60 \mathrm{~cm}$} & \multicolumn{3}{|c|}{$60-90 \mathrm{~cm}$} & \multicolumn{2}{|c|}{$\sum 0-90 \mathrm{~cm}$} \\
\hline & & & & & \multicolumn{2}{|c|}{$\mathrm{C}_{\text {org }}$-Vorrat } & \multirow[t]{2}{*}{$\mathbf{n}$} & \multicolumn{2}{|c|}{$\mathrm{C}_{\text {org }}$-Vorrat } & \multirow[t]{2}{*}{$\mathbf{n}$} & \multicolumn{2}{|c|}{$\mathrm{C}_{\text {org }}$-Vorrat } & \multirow[t]{2}{*}{$\mathbf{n}$} & \multicolumn{2}{|c|}{$\mathrm{C}_{\text {org }}$-Vorrat } \\
\hline & & & & & $\left(\mathrm{tha}^{-1}\right)$ & (Mt) & & $\left(\mathrm{tha}^{-1}\right)$ & (Mt) & & $\left(\mathrm{tha}^{-1}\right)$ & (Mt) & & $\left(\mathbf{t} \mathbf{h a}^{-1}\right)$ & (Mt) \\
\hline $\begin{array}{c}\text { Pseudogley- } \\
\text { Parabraunerde }\end{array}$ & 18,6 & 5,5 & 23 & 153.662 & 62 & 9,5 & 26 & 17 & 2,6 & 27 & 11 & 1,7 & 26 & 90 & 13,8 \\
\hline Pseudogley & 20,9 & 13,3 & 20 & 134.791 & 79 & 10,7 & 19 & 19 & 2,6 & 24 & 9 & 1,3 & 24 & 108 & 14,6 \\
\hline Kolluvisol & 12,4 & 9,2 & 16 & 106.485 & 85 & 9,0 & 13 & 71 & 7,6 & 11 & 49 & 5,2 & 14 & 205 & 21,8 \\
\hline Braunerde & 4,9 & 3,3 & 12 & 81.548 & 55 & 4,5 & 21 & 15 & 1,2 & 26 & 7 & 0,5 & 32 & 77 & 6,3 \\
\hline Parabraunerde & 15,4 & 4,8 & 8 & 50.547 & 52 & 2,6 & 22 & 18 & 0,9 & 26 & 9 & 0,5 & 28 & 79 & 4,0 \\
\hline Niedermoor & & & 6 & 37.741 & 455 & 17,2 & 1 & 204 & 7,7 & 5 & 175 & 6,6 & 6 & 835 & 31,5 \\
\hline $\begin{array}{c}\text { Pseudogley- } \\
\text { Braunerde }\end{array}$ & 9,1 & 4,5 & 4 & 26.958 & 60 & 1,6 & 7 & 17 & 0,5 & 5 & 6 & 0,2 & 7 & 82 & 2,2 \\
\hline Summe & & & 88 & 591.732 & & 55 & 109 & & 23 & 124 & & 16 & 137 & & 94 \\
\hline sonstige & & & 12 & 82.222 & & & & & & & & & & & \\
\hline
\end{tabular}

Vorgeest

\begin{tabular}{ccccc} 
& \multicolumn{4}{c}{} \\
\cline { 2 - 5 } Bodentyp & Ton $(\mathbf{x})$ & $\begin{array}{c}\text { Ton } \\
(+/-)\end{array}$ & $\begin{array}{c}\text { Rel. } \\
\text { Fläche }\end{array}$ & $\begin{array}{c}\text { Abs. } \\
\text { Fläche }\end{array}$ \\
& $(\%)$ & $(\%)$ & $(\%)$ & $(\mathbf{h a})$ \\
\hline Gley-Podsol & 2,9 & 1,0 & 36,3 & 72.954 \\
Gley & 6,9 & 5,8 & 13,6 & 27.333 \\
Braunerde-Podsol & 3,7 & 1,4 & 12,4 & 24.921 \\
Braunerde & 3,7 & 1,6 & 11,2 & 22.509 \\
Podsol & 2,6 & 1,3 & 8 & 16.078 \\
Niedermoor & - & - & 6,1 & 12.259 \\
Hochmoor & - & - & 3,7 & 7436 \\
\hline Summe & & & 91 & 183.490 \\
sonstige & & & 9 & 17.485
\end{tabular}

\begin{tabular}{ccc}
\multicolumn{3}{c}{$\mathbf{0 - 3 0} \mathbf{~ c m}$} \\
\hline $\mathbf{C}_{\text {org }}$-Vorrat & $\mathbf{n}$ \\
$\left(\mathbf{t ~ h a}^{-1}\right)$ & $(\mathbf{M t})$ & \\
\hline 137 & 10,0 & 16 \\
120 & 3,3 & 6 \\
74 & 1,8 & 3 \\
86 & 1,9 & 7 \\
66 & 1,1 & 8 \\
311 & 3,8 & 5 \\
242 & 1,8 & 3 \\
\hline & 24 & 48 \\
& &
\end{tabular}

\begin{tabular}{ccc}
\multicolumn{3}{c}{$\mathbf{3 0 - 6 0} \mathbf{~ c m}$} \\
\hline $\mathbf{C}_{\text {org }}$-Vorrat & $\mathbf{n}$ \\
$\left.\mathbf{( t ~ h a}^{-1}\right)$ & $(\mathbf{M t})$ & \\
\hline 60 & 4,4 & 16 \\
27 & 0,7 & 9 \\
26 & 0,6 & 4 \\
21 & 0,5 & 12 \\
28 & 0,5 & 13 \\
214 & 2,6 & 10 \\
179 & 1,3 & 6 \\
\hline & 11 & 70 \\
& &
\end{tabular}

\begin{tabular}{|c|c|c|}
\hline $60-90 \mathrm{~cm}$ & & $\sum 0-90 \mathrm{~cm}$ \\
\hline
\end{tabular}

Hohe Geest

\begin{tabular}{ccccc}
\hline Bodentyp & Ton (x) & $\begin{array}{c}\text { Ton } \\
(+/-)\end{array}$ & $\begin{array}{c}\text { Rel. } \\
\text { Fläche }\end{array}$ & $\begin{array}{c}\text { Abs. } \\
\text { Fläche }\end{array}$ \\
& $\mathbf{( \% )}$ & $(\%)$ & $(\%)$ & $(\mathbf{h a})$ \\
\hline Braunerde & 4,5 & 2,0 & 18,2 & 68.535 \\
Pseudogley & 15,5 & 8,8 & 11,9 & 44.811 \\
Gley-Podsol & 3,7 & 1,4 & 10 & 37.657 \\
Pseudogley- & 6,1 & 2,4 & 8,8 & 33.138 \\
Braunerde & 4,5 & 1,9 & 8,7 & 32.761 \\
Braunerde-Podsol & 9,2 & 5,6 & 6,7 & 25.230 \\
Gley & 7,9 & 1,5 & 6,2 & 23.347 \\
Kolluvisol & - & - & 4,8 & 18.075 \\
Niedermoor & 7,3 & 2,6 & 4,7 & 17.699 \\
Pseudogley-Podsol & 3,8 & 2,0 & 4,5 & 16.945 \\
Podsol & 7,5 & - & 4,3 & 16.192 \\
Plaggenesch & 11,8 & 0,3 & 3,4 & 12.803 \\
Parabraunerde & 19.193 \\
\hline Summe & & & 92 & 347.193 \\
sonstige & & & 8 & 29.372
\end{tabular}

\begin{tabular}{ccc}
\multicolumn{3}{c}{$0-30 \mathrm{~cm}$} \\
\hline $\mathbf{C}_{\text {org }}$-Vorrat & $\mathbf{n}$ \\
$\left(\mathbf{t ~ h a}^{-1}\right)$ & $(\mathbf{M t})$ & \\
\hline 79 & 5,4 & 33 \\
93 & 4,2 & 51 \\
138 & 5,2 & 16 \\
63 & 2,1 & 8 \\
96 & 3,1 & 7 \\
108 & 2,7 & 10 \\
65 & 1,5 & 4 \\
307 & 5,6 & 11 \\
154 & 2,7 & 10 \\
97 & 1,6 & 24 \\
92 & 1,5 & 1 \\
61 & 0,8 & 2 \\
\hline & 36 & 177 \\
& &
\end{tabular}

\begin{tabular}{ccc}
\multicolumn{3}{c}{$30-60 \mathrm{~cm}$} \\
\hline $\mathbf{C}_{\text {org }}$-Vorrat & $\mathbf{n}$ \\
$\left(\mathbf{t ~ h a}^{-1}\right)$ & $(\mathbf{M t})$ & \\
\hline 19 & 1,3 & 34 \\
26 & 1,2 & 49 \\
66 & 2,5 & 15 \\
20 & 0,7 & 6 \\
23 & 0,7 & 11 \\
31 & 0,8 & 10 \\
31 & 0,7 & 4 \\
204 & 3,7 & 14 \\
49 & 0,9 & 7 \\
37 & 0,6 & 24 \\
65 & 1,1 & 1 \\
23 & 0,3 & 2 \\
\hline & 14 & 177
\end{tabular}

\begin{tabular}{|c|c|c|c|c|}
\hline \multicolumn{3}{|c|}{$60-90 \mathrm{~cm}$} & \multicolumn{2}{|c|}{$\sum 0-90 \mathrm{~cm}$} \\
\hline \multicolumn{2}{|c|}{$\mathrm{C}_{\text {org }}$-Vorrat } & \multirow[t]{2}{*}{ n } & \multicolumn{2}{|c|}{$\mathrm{C}_{\text {org }}$-Vorrat } \\
\hline$\left(\mathbf{t h a}^{-1}\right)$ & (Mt) & & $\left(\mathrm{tha}^{-1}\right)$ & (Mt) \\
\hline 8 & 0,5 & 35 & 106 & 7,3 \\
\hline 11 & 0,5 & 54 & 130 & 5,8 \\
\hline 18 & 0,7 & 19 & 223 & 8,4 \\
\hline 12 & 0,4 & 8 & 96 & 3,2 \\
\hline 9 & 0,3 & 11 & 127 & 4,2 \\
\hline 18 & 0,4 & 14 & 157 & 4,0 \\
\hline 14 & 0,3 & 3 & 111 & 2,6 \\
\hline 173 & 3,1 & 18 & 684 & 12,4 \\
\hline 11 & 0,2 & 11 & 214 & 3,8 \\
\hline 14 & 0,2 & 27 & 148 & 2,5 \\
\hline 21 & 0,3 & 1 & 178 & 2,9 \\
\hline \multirow[t]{2}{*}{11} & 0,1 & 2 & 94 & 1,2 \\
\hline & 7 & 203 & & 58 \\
\hline
\end{tabular}


Tabelle 1. Fortsetzung. Tiefenspezifische organische Kohlenstoffvorräte $\left(\mathrm{C}_{\text {orr }}\right)$ in 0-90 cm Bodentiefe von repräsentativen Bodentypen mit einem relativen Flächenanteil von $>3 \%$ in den Hauptnaturräumen Schleswig-Holsteins. $\mathrm{n}=$ Anzahl der Bodenprofile, $\mathrm{x}=$ arithmetischer Mittelwert, +/- = Standardabweichung, Datengrundlage: LLUR.

Table 1. Continuation. Depth-specific soil organic carbon (SOC) stocks from $0-90 \mathrm{~cm}$ depth for the most frequent soil types with a relative area $>3 \%$ in the different geological regions in Schleswig-Holstein. $\mathrm{n}=$ number of soil profiles, $\mathrm{x}=$ arithmetic mean, $+/-=$ standard deviation, data basis: LLUR.

Marsch

\begin{tabular}{|c|c|c|c|c|c|c|c|}
\hline \multirow{3}{*}{ Bodentyp } & \multirow{3}{*}{$\begin{array}{c}\text { Ton }(\mathbf{x}) \\
(\%)\end{array}$} & \multirow{3}{*}{$\begin{array}{l}\text { Ton } \\
(+/-) \\
(\%)\end{array}$} & \multirow{3}{*}{$\begin{array}{c}\text { Rel. } \\
\text { Fläche } \\
(\%)\end{array}$} & \multirow{3}{*}{$\begin{array}{l}\text { Abs. } \\
\text { Fläche } \\
\text { (ha) }\end{array}$} & \multicolumn{3}{|c|}{$0-30 \mathrm{~cm}$} \\
\hline & & & & & \multicolumn{2}{|c|}{$\mathrm{C}_{\text {org }}$-Vorrat } & $\mathbf{n}$ \\
\hline & & & & & $\left(\mathrm{t} \mathrm{ha}^{-1}\right)$ & (Mt) & \\
\hline Kleimarsch & 25,8 & 7,7 & 27,0 & 77.048 & 69 & 5,3 & 20 \\
\hline Dwogmarsch & 36,8 & 7,7 & 17,6 & 50.224 & 70 & 3,5 & 19 \\
\hline Kalkmarsch & 15,8 & 7,2 & 16,8 & 47.941 & 51 & 2,5 & 36 \\
\hline Knickmarsch & 42,5 & 8,6 & 8,2 & 23.400 & 100 & 2,3 & 4 \\
\hline Niedermoor & - & - & 6,7 & 19.119 & 259 & 5,0 & 1 \\
\hline Rohmarsch & 18,6 & 11,0 & 4,5 & 12.841 & 58 & 0,7 & 7 \\
\hline Hochmoor & - & - & 4,0 & 11.415 & 285 & 3,3 & 6 \\
\hline Organomarsch & 37,5 & 16,5 & 3,0 & 8276 & 198 & 1,6 & 3 \\
\hline Summe & & & 88 & 250.264 & & 24 & 96 \\
\hline sonstige & & & 12 & 35.100 & & & \\
\hline
\end{tabular}

\begin{tabular}{ccc}
\hline \multicolumn{3}{c}{$\mathbf{3 0 - 6 0 ~} \mathbf{~ c m}$} \\
$\mathbf{C}_{\text {org }}$-Vorrat & $\mathbf{n}$ \\
$\left(\mathbf{t ~ \mathbf { h a } ^ { - 1 } )}\right.$ & $\mathbf{( M t )}$ & \\
\hline 32 & 2,4 & 40 \\
38 & 1,9 & 39 \\
25 & 1,2 & 47 \\
53 & 1,2 & 5 \\
223 & 4,3 & 2 \\
34 & 0,4 & 9 \\
177 & 2,0 & 6 \\
207 & 1,7 & 6 \\
\hline & 15 & 154
\end{tabular}

\begin{tabular}{|c|c|c|}
\hline \multicolumn{2}{|c|}{$60-90 \mathrm{~cm}$} & \multirow[t]{2}{*}{$\mathbf{n}$} \\
\hline$\left(\mathbf{t} \mathbf{h a}^{-1}\right)$ & (Mt) & \\
\hline 23 & 1,8 & 43 \\
\hline 31 & 1,5 & 45 \\
\hline 20 & 0,9 & 50 \\
\hline 39 & 0,9 & 7 \\
\hline 192 & 3,7 & 2 \\
\hline 36 & 0,5 & 14 \\
\hline 230 & 2,6 & 5 \\
\hline \multirow[t]{2}{*}{88} & 0,7 & 7 \\
\hline & 13 & 173 \\
\hline
\end{tabular}

Hohe $\mathrm{C}_{\text {org }}$-Vorräte sind in den B-Horizonten podsolierter Böden, der (Gley-)Podsole der Vorgeest und Hohen Geest vorzufinden. Knapp Dreiviertel $(71 \%)$ des in der Vorgeest gespeicherten Kohlenstoffs sind in den stark verbreiteten Gley-Podsolen (36 \% Flächenanteil) und in den Hoch- und Niedermoorböden $(<6 \%$ Flächenanteil) wiederzufinden (Abbildung 1). In der Hohen Geest sind zusätzlich zu den Niedermooren und (Gley-)Podsolen auch Pseudogley-Podsole und die in Schleswig-Holstein im Unterschied zu Niedersachsen nur mit einem geringen Flächenanteil vorkommenden Plaggenesche mit erhöhten
$\mathrm{C}_{\text {org }}$-Vorräten ( $>170 \mathrm{t} / \mathrm{ha}$ ) vertreten. Sie tragen damit zu $5 \%$ des Gesamt- $\mathrm{C}_{\text {org }}$-Vorrats der Hohen Geest bei (Abbildung 1). Mehr als $50 \%$ der Fläche werden dagegen von terrestrischen Böden eingenommen, deren durchschnittliche $\mathrm{C}_{\text {org }}$-Vorräte mit Ausnahme der Podsole weniger als $130 \mathrm{t} /$ ha bis in $90 \mathrm{~cm}$ Bodentiefe betragen.

Hinsichtlich der tiefenspezifischen $\mathrm{C}_{\text {org }}$-Verteilung ist festzustellen, dass in den Böden der Jungmoränenlandschaft nahezu $60 \%$ des $\mathrm{C}_{\text {org }}$-Vorrats in den obersten $30 \mathrm{~cm}$ und weitere $25 \%$ in der Tiefe bis $60 \mathrm{~cm}$ gespeichert sind. Eine vergleichbare Verteilung findet man auch in der Vorgeest und
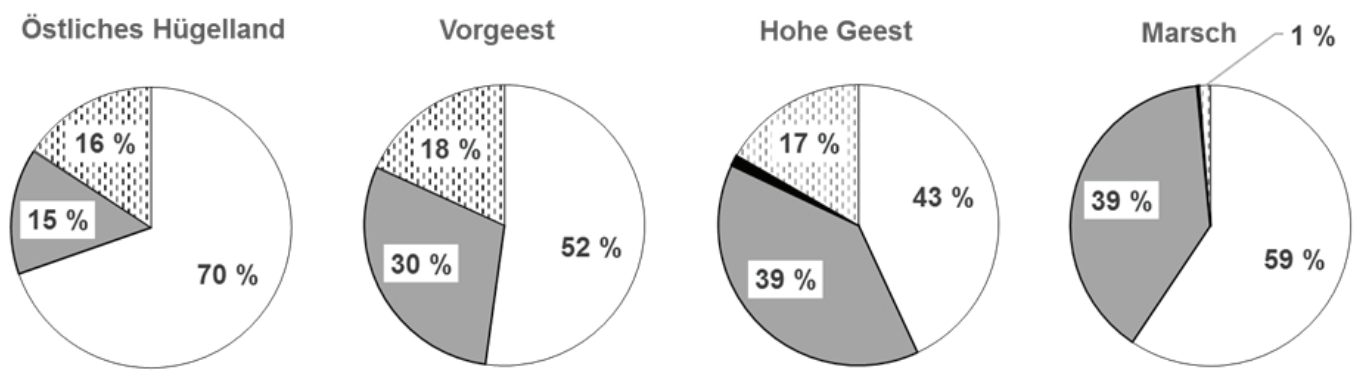

$\square$ Ackerland

口Dauergrünland

- Sonstige

๑Wald

Abbildung 2. Prozentualer Anteil der 3 dominierenden Landnutzungssysteme (Ackerland, Dauergrünland und Forst) an der gesamten land- und forstwirtschaftlich genutzten Fläche in Schleswig-Holstein. Eigene Darstellung; Datengrundlage: DESTATIS (2015) und MELUND (2018). Figure 2. Relative share of the 3 main land use management systems (arable land, permanent grassland and forest) on the total agricultural and forestry area in Schleswig-Holstein Authors' own representation; data source: DESTATIS (2015) und MELUND (2018). 


\section{- $\mathrm{C}_{\text {org }}$ Gehalt $\square \mathrm{C}_{\text {org }}$-Vorrat (0-30 cm Tiefe)}
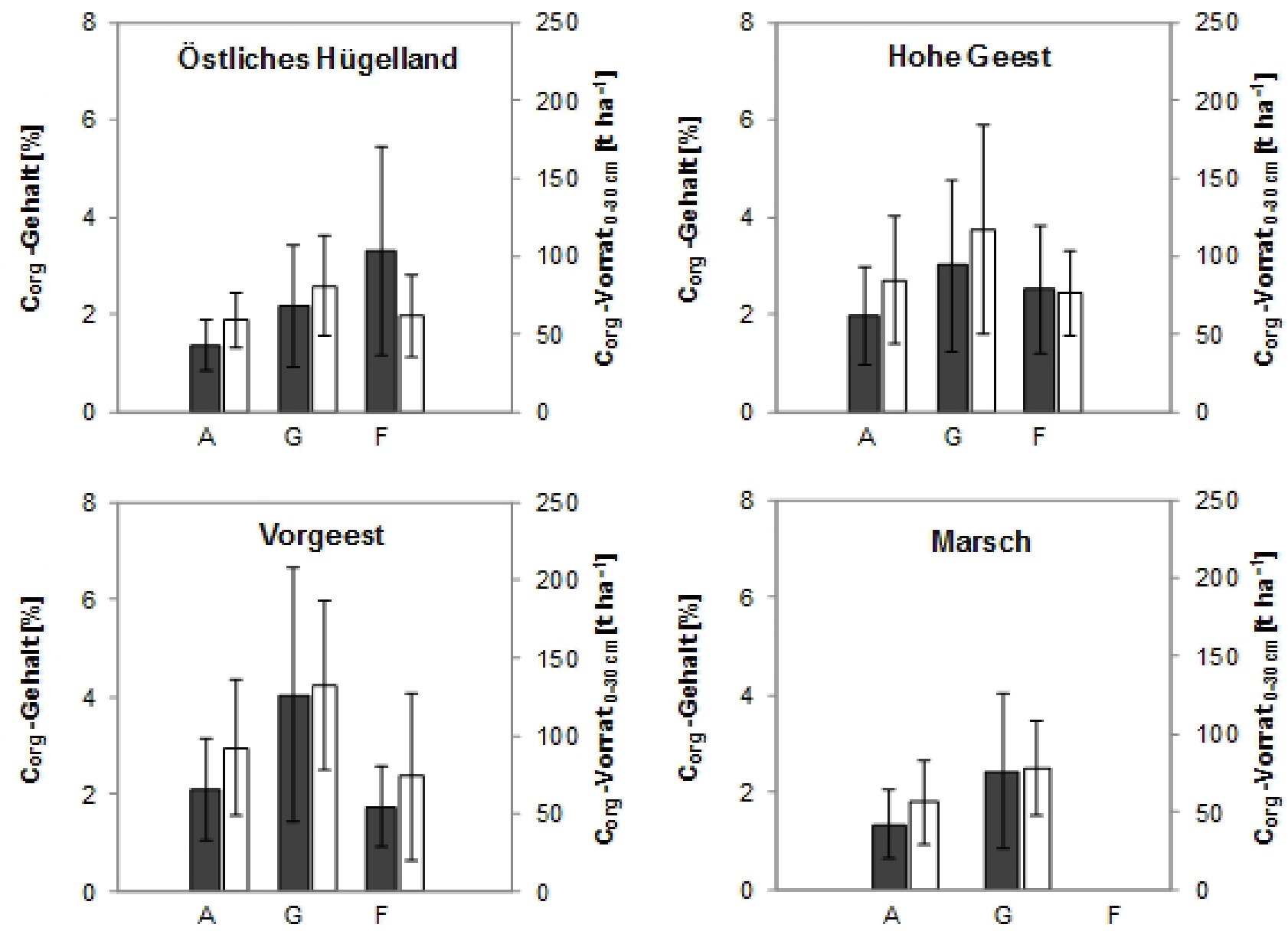

Abbildung 3. Einfluss des Landnutzungstyps $(A=$ Ackerland, $G=$ Grünland, $F=$ Forst $)$ auf den mittleren organischen Kohlenstoff $\left(\mathrm{C}_{\text {org }}\right)$-Gehalt mineralischer A-Horizonte und $\mathrm{C}_{\mathrm{org}}$-Vorräte in 0-30 cm Bodentiefe von repräsentativen Bodentypen der Hauptnaturräume Schleswig-Holsteins. Fehlerbalken zeigen die arithmetische Standardabweichung an. Repräsentative Bodentypen und Anzahl der Bodenprofile sind Tabelle 1 zu entnehmen. Organische Böden sind in der Darstellung nicht enthalten.

Figure 3. Effect of land use management $(A=$ Arable land, $G=$ Grassland, $F=$ Forest $)$ on mean soil organic carbon (SOC) content of mineral A-horizons and SOC stock within 0-30 cm depths for the region-specific soil types in Schleswig-Holstein. Error bars indicate arithmetic standard deviation. Representative soil types for each geological region and corresponding number of soil profiles are given in Table 1. Organic soils are excluded.

in der Hohen Geest, während in der Marsch eine weitgehend gleichmäßige $\mathrm{C}_{\text {org }}$-Verteilung aufgrund der bereits im meeresbürtigen Sediment vorhandenen organischen Bestandteile (sogenannte primäre organische Substanz) nachgewiesen werden kann. Somit verfügen die Marschen von Natur aus über sehr hohe $\mathrm{C}_{\text {org }}$-Vorräte über die gesamte Tiefe. Neben den Mooren sind hier die Organomarschen mit ihren stark humosen Unterböden hervorzuheben, die mit knapp 500 t/ha bis $90 \mathrm{~cm}$ Tiefe sehr hohe $\mathrm{C}_{\text {org }}$-Vorräte aufweisen. Aufgrund ihrer geringen Flächenverbreitung (3\%) decken sie allerdings nur $8 \%$ des gesamten $\mathrm{C}_{\text {org }}$-Vorrats der Marschen ab.

\subsection{Einfluss der Landnutzung auf die $\mathrm{C}_{\mathrm{org}}$-Vorräte}

Während 60-70 \% der sehr fruchtbaren Böden im Östlichen Hügelland und in der Marsch ackerbaulich bewirtschaftet werden, findet auf den weniger fruchtbaren Böden der Geest auf 30-40\% Grünland- und auf knapp $20 \%$ Waldnutzung statt (Abbildung 2).

Landnutzungsspezifische Unterschiede in der $\mathrm{C}_{\text {org }}$-Speicherung in Böden werden über die $\mathrm{C}_{\text {org }}$-Gehalte der A-Horizonte sowie den berechneten $\mathrm{C}_{\text {org }}$-Vorräten in 0-30 cm Tiefe deutlich (Abbildung 3). In allen vier Hauptnatur- 
räumen weisen die als Grünland genutzten Oberböden stets die höchsten $\mathrm{C}_{\text {org }}$-Vorräte auf, wobei in den Böden der Vorgeest und Hohen Geest mit bis zu ca. 130 t/ha die höchsten Vorräte in den oberen $30 \mathrm{~cm}$ festzustellen sind. Deutlich geringere $\mathrm{C}_{\text {org }}$-Vorräte werden in den ackerbaulich genutzten Flächen $(<100 \mathrm{t} / \mathrm{ha})$ und den Wäldern $(<80 \mathrm{t} / \mathrm{ha})$ in allen vier Hauptnaturräumen gespeichert. Insbesondere in den Ackerbauregionen Östliches Hügelland und Marsch treten die geringsten $\mathrm{C}_{\text {org }}$-Vorräte $<60 \mathrm{t} /$ ha im gepflügten Oberboden auf. Es ist jedoch darauf hinzuweisen, dass für die Berechnung der $\mathrm{C}_{\text {org }}$-Gesamtmengen je nach Landnutzungstyp eine unterschiedliche Verteilung der Bodentypen zugrunde liegt und dass unter Grünlandnutzung eine höhere Anzahl hydromorpher Böden (u. a. Gleye, Anmoorgleye) vertreten ist als unter Ackernutzung, da die Nutzungsart meistens an die Standortverhältnisse (insbesondere an den Grundwasserspiegel) gebunden ist. Unter Waldböden ist festzustellen, dass hohe $\mathrm{C}_{\text {org }}$-Gehalte des A-Horizontes nicht zwangsläufig mit hohen Vorräten in $0-30 \mathrm{~cm}$ Tiefe verbunden sind (siehe Östliches Hügelland), was im Wesentlichen auf die geringe Mächtigkeit der Ah-Horizonte unter Wald im Vergleich zu den anderen Landnutzungsformen zurückzuführen ist. Die $\mathrm{C}_{\text {org }}$-Vorräte der Waldböden im Östlichen Hügelland sind dabei im Vergleich zu denen in der Vorgeest und Hohen Geest um ca. 10 t/ha geringer. Hier ist zu berücksichtigen, dass die C-Mengen aus organischen Auflagen nicht in die Berechnung eingegangen sind.

\section{Diskussion}

Humus ist nicht nur für die Nährstoffsorption und verbesserte Wasserspeicherung sowie für eine erhöhte mechanische Stabilisierung der Böden von Bedeutung, sondern führt zu einer entsprechenden Entlastung der Atmosphäre hinsichtlich der $\mathrm{CO}_{2}$-Emissionen. Diese positiven Aspekte unterliegen allerdings durchaus kurzzeitigen Schwankungen, denn der Humusauf- und -abbau wird neben den entscheidenden Standortfaktoren wie u. a. der Hydromorphie, dem Mikroklima und der biologischen Aktivität auch von der Landnutzung ( $\mathrm{Zu}$ - und Abfuhr von organischer Substanz/Biomasse, Bodenbearbeitung, Be- und Entwässerungsmaßnahmen) beeinflusst.

In den vier Hauptnaturräumen in Schleswig-Holstein weisen die Grünlandstandorte im Vergleich zu den Acker- und Waldstandorten die höchsten Humusvorräte im Oberboden (0-30 cm unter GOF) auf (Abbildung 3). Hier ist zu beachten, dass es sich um einen Status quo der Humusvorräte in Böden unter den drei Landnutzungsformen Ackerland, Grünland und Wald in Schleswig-Holstein handelt, der bisher weder die zeitliche Veränderung der Humusvorräte noch die jeweiligen Standortgegebenheiten berücksichtigt. So findet die Grünlandnutzung vorzugsweise auf feuchteren Standorten (hydromorphen Böden) statt, die aufgrund des Wassereinflusses und der dadurch reduzierten Mineralisation der organischen Substanz bereits von Natur aus über erhöhte Humusgehalte (primär oder sekundär angereicherte organische Substanz) verfügen, während anhydromorphe Böden vorzugsweise für den Ackerbau genutzt werden. Hinzu kommt, dass feuchtere Standorte durch Dränung ackerbaulich nutzbar gemacht wurden, wodurch die zunehmende Belüftung zu einem verstärkten Humusabbau geführt hat. Der anthropogen bedingte Landnutzungseinfluss auf die Humusspeicherung steht dabei in Wechselwirkung mit der oben genannten standortspezifischen Humusanreicherung, wodurch der anthropogene Effekt der Grünlandnutzung überschätzt sein könnte.

Das bedeutet gleichzeitig, dass das Speicher- und Verlustpotenzial von Humusmengen durch einen Landnutzungswechsel (z. B. Acker- zu Grünland und umgekehrt) bodentypspezifisch zu bewerten ist (Tabelle 1). Dies betrifft auch die Humusverluste nach einem Grünlandumbruch, die in Abhängigkeit des Standortes (Bodentyp, Tongehalt, Klima) unterschiedlich hoch ausfallen, wobei mit Spannbreiten von 20-80 \% zu rechnen ist (Springob et al., 2001; Freibauer et al., 2004; Poeplau et al., 2011; Lugato et al., 2014; LfULG, 2015). Bezogen auf den Oberboden $(0-30 \mathrm{~cm}$ ) kann damit ein hoher Anteil (ca. $60 \%$, vgl. auch Waldmann und Weinzierl, 2014) der $\mathrm{C}_{\text {org }}$-Vorräte durch eine standortangepasste Landnutzung (u. a. Vegetation, Bodenbearbeitung) beeinflusst werden. Vor diesem Hintergrund sind auch die bekannten Vorteile einer Minimal- Bodenbewirtschaftung erneut zu diskutieren, da eine erhöhte Freisetzung von Kohlenstoff aus dem Aggregatverband durch den Verzicht der Pflugarbeit reduziert werden kann (Six et al., 2002; Mordhorst et al., 2013; Wiesmeier et al., 2014).

Im Gegensatz dazu ist die $\mathrm{C}_{\text {org }}$-Speicherung im Unterboden stärker von geogenen sowie pedogenen Eigenschaften der Böden geprägt, sodass sich bodentypspezifische Unterschiede im $\mathrm{C}_{\text {org }}$-Vorrat herausstellen. Während das meeresbürtige Ausgangssediment der Marschböden bereits über hohe, primäre $\mathrm{C}_{\text {org }}$-Gehalte verfügt, beruhen die $\mathrm{C}_{\text {org }}$-Gehalte im Unterboden der (Gley-)Podsole in 
der Vor- und Hohen Geest auf Verlagerungsprozessen von metallorganischen Verbindungen. Bei der Bewertung der Humusqualität ist allerdings zu berücksichtigen, dass die Nährstoffverfügbarkeit (weites C/N-Verhältnis und die Erreichbarkeit für Pflanzenwurzeln) in den z. T. verkitteten Bhs-Horizonten (Orterde und Ortstein) (Wiechmann, 1995) im Vergleich zu den humusreichen Unterböden der Marsch mit engem C/N-Verhältnis gering ist (Janetzko \& Fleige, 1995).

Vergleicht man die in den schleswig-holsteinischen Böden gespeicherten $\mathrm{C}_{\text {org }}$-Mengen mit Daten anderer Bundesländer, so finden sich in Abhängigkeit von der Landnutzung vergleichbare Größenordnungen: Wiesmeier et al. (2012) haben die Daten für Bayern auf der Grundlage von 1500 Bodenprofilen ausgewertet. Auch sie finden auf Grünlandstandorten die höchsten $\mathrm{C}_{\text {org }}$-Vorräte bis in $1 \mathrm{~m}$ Bodentiefe mit einem mittleren Wert von $118 \mathrm{t} / \mathrm{ha}$, während geringere Vorräte von 90-98 t/ha in Acker- und Waldböden errechnet worden sind.

Werden nur die am stärksten von der Landnutzung beeinflussten $0-30 \mathrm{~cm}$ betrachtet, wird deutlich, dass die $\mathrm{C}_{\text {org }}$-Vorräte bis zu ca. $130 \mathrm{t} / \mathrm{ha}$ in $0-30 \mathrm{~cm}$ Tiefe auf den Grünlandstandorten der Geest (ohne Berücksichtigung der Moore) im Vergleich zu denen in Wiesmeier et al. (2012) aufgeführten europäischen Böden $(<95 \mathrm{t} / \mathrm{ha}$ für 0-30 cm Tiefe) sogar deutlich höher liegen. Dagegen sind die geringeren $\mathrm{C}_{\text {org }}$-Vorräte $(60 \mathrm{t} / \mathrm{ha})$ in den Oberböden des Östlichen Hügellands und der Marsch mit denen aus Baden-Württemberg vergleichbar (Neufeldt, 2005). Eine Studie aus der Schweiz zeigt in Bezug auf die Landnutzung gleiche Tendenzen in den Humusvorräten, allerdings mit geringeren Unterschieden zwischen Ackerland (54 t/ha) und Dauergrünland (64 t/ha für 0-30 cm Tiefe) (Leifeld et al., 2005). In Bezug auf die Waldnutzung stimmen die $\mathrm{C}_{\text {org }}$-Vorräte von durchschnittlich $70 \mathrm{t} / \mathrm{ha}(0-30 \mathrm{~cm}$ Tiefe $)$ mit denen von Wördehoff et al. (2012) für Geschiebesande und -lehme in Schleswig-Holstein überein, während höhere Vorräte in schwach verlehmten Sandböden vorgefunden wurden, die vorwiegend mit Podsolierungsprozessen in Verbindung standen (Wördehoff et al., 2012). Vergleichsweise höhere $\mathrm{C}_{\text {org }}$-Vorräte (> $70 \mathrm{t} / \mathrm{ha}$ ) in den Oberböden der Vorgeest und Hohen Geest mit einem hohen Anteil podsolierter Böden (siehe Tab. 1) zeigt ebenfalls Abbildung 3.

Auf die gesamte Fläche Schleswig-Holsteins bezogen ist festzustellen, dass im Vergleich zu anderen Bundesländern Deutschlands relativ hohe Humusvorräte bis in $90 \mathrm{~cm}$ Tiefe vorhanden sind. Der Gesamtvorrat von $244 \mathrm{Mt} \mathrm{C}_{\text {org }}$ übersteigt beispielsweise den von Sachsen-Anhalt mit den dort verbreiteten humusreichen Schwarzerden (185 Mt bei ähnlicher Flächengröße) und liegt nicht weit unter dem Gesamtvorrat des Bundeslandes Baden-Württemberg (342 $\mathrm{Mt} \mathrm{C}_{\text {org }}$ ), das eine im Vergleich zu Schleswig-Holstein mehr als doppelt so hohe Flächengröße, jedoch geringere bzw. keine Vorkommen von Mooren bzw. Marschen aufweist (LAGB, 2014; Waldmann und Weinzierl, 2014). Während die Erhaltung der Moore in Schleswig-Holstein als größte C-Senke (siehe Abbildung 1) und die Nutzung grundwassernaher Standorte, der hydromorphen Bodentypen (Gleye, Anmoorgleye), als Dauergrünland bereits zu den hohen $\mathrm{C}_{\text {org }}$-Vorräten beitragen, variieren die $\mathrm{C}_{\text {org }}$ Vorräte und das Humussteigerungspotenzial unter Ackernutzung in Abhängigkeit der Standortgegebenheiten (u. a. Textur und ertragswirksame Faktoren wie pH-Wert, Nährund Wasserhaushalt des Bodens). Folgt man den von Schiefer et al. (2015) entwickelten Intensivierungspotenzialen, sind die fruchtbaren Ackerstandorte aus der Marsch und dem Östlichen Hügelland aufgrund ihrer verbesserten Nährstoffverfügbarkeit und feinkörnigeren Textur (hoher Ton- und Schluffanteil) im Vergleich zu sandigen Geeststandorten auch als Standorte mit hohem zusätzlichen C-Sequestrierungspotenzial anzusehen. Dabei ist zu berücksichtigen, dass nach Schiefer et al. (2015) eine Intensivierung des Ackerbaus (höherer Ertrag pro Flächeneinheit) eine nachhaltige und umweltverträgliche Landbewirtschaftung (geringere Umweltbelastung pro Ertragseinheit) voraussetzt, die daher nur durch eine flächendeckendere Umstellung auf konservierende Verfahren (wie pfluglose oder reduzierte Bodenbearbeitung, Rückführung von Ernterückständen oder Gärresten, Zwischenfruchtanbau, Futtergräser/Leguminosen als Fruchtfolgeglieder (Lugato et al., 2014) auf Ackerböden gewährleistet werden kann.

\section{Schlussfolgerung}

Der Humusvorrat in schleswig-holsteinischen Böden variiert in Abhängigkeit von den in den Hauptnaturräumen vorliegenden Ausgangsgesteinen und den Bodentypen sowie der Landnutzung. In Bezug auf den gesamten (absoluten) $\mathrm{C}_{\text {org }}$-Vorrat bis $90 \mathrm{~cm}$ Tiefe der vier Hauptnaturräume Schleswig-Holsteins steigen die $\mathrm{C}_{\text {org }}$-Mengen der Böden mit der Flächengröße der Hauptnaturräume an. Die größten Humusmengen verzeichnet daher das Östliche Hügelland, während der flächenmäßig kleinste Hauptnaturraum (Vorgeest) die geringsten Mengen aufweist. Betrachtet 
man das C-Speicherungsvermögen der Hauptnaturräume jedoch pro Flächeneinheit, ist der durchschnittliche $\mathrm{C}_{\text {org }}$ Vorrat ( $t / h a)$ für Böden in der Marsch am größten und nimmt in der Reihenfolge Marsch > Vorgeest > Östliches Hügelland > Hohe Geest ab. Große C-Pools stellen hinter den Hoch- und Niedermoorböden folgend die stark verbreiteten Gley-Podsole (Hohe Geest und Vorgeest) und die gering verbreiteten Organomarschen dar, was im Alt- und Jungmoränengebiet (Hohe Geest und Östliches Hügelland) für die Kolluvisole zutrifft.

In Abhängigkeit von den Standorteigenschaften (Bodentyp und geologischem Ausgangsgestein) ergeben sich so unterschiedliche C-Sequestrierungspotenziale, die im Hinblick auf Strategien zur Humussteigerung in den Böden bzw. zur verringerten Freisetzung klimarelevanter Gase $\left(\mathrm{CO}_{2}\right.$, $\mathrm{N}_{2} \mathrm{O}$ ) durch eine standortangepasste und bodenschonende Landbewirtschaftung (z. B. reduzierte Bodenbearbeitung) zu berücksichtigen sind.

\section{Literatur}

$4 p 1000$ (2017): „4 per 1000” Initiative: Carbon sequestration in soils for food security and the climate. www.4p1000.org.

Balesdent, J. und D. Arrouays (1999): Usage des terres et stockage de carbone dans les sols du territoire francais. Une estimation des flux nets annuels pour la période 1900-1999. Comptes rendus de l'Académie d'Agriculture de France 85, 265-277.

Batjes, N.H. (2016): Harmonized soil property values for broad-scale modelling (WISE30sec) with estimates of global soil carbon stocks. Geoderma 269, 61-68.

Blume, H.-P., Brümmer, G.W., Fleige, H., Horn, R., Kandeler, E., Kögel-Knabner, I., Kretzschmar, R., Stahr, K. und B.M. Wilke (2010): Scheffer/Schachtschabel: Lehrbuch der Bodenkunde. 16. Aufl., Spektrum Akademischer Verlag, Heidelberg, Deutschland.

Blume, H.-P., Stahr, K. und P. Leinweber (2011): Bodenkundliches Praktikum. 3. Aufl., Spektrum Akademischer Verlag, Heidelberg, Deutschland.

Chen, S., Martin, M.P., Saby, N.P.A., Walter, C., Angers, D.A. und D. Arrouays (2018): Fine resolution map of top- and subsoil carbon sequestration potential in France. Science of the Total Environment 60, 389-400. Chenu, C., Angers, D., Barré, P., Derrien, D., Arrouays, D. und J. Balesdent (2018): Increasing organic stocks in agricultural soils: knowledge gaps and potential in- novations. Soil and Tillage Research, doi.org/10.1016/j. still.2018.04.011 (in press).

Conrad, K.A., Dalal, R.C., Dalzell, S.A., Allen, D.E. und N.W. Menzies (2017): The sequestration and turnover of soil organic carbon in subtropical leucaena-grass pastures. Agriculture Ecosystems \& Environment 248, 38-47.

Cosentino, D., Le Bissonnais, Y. und C. Chenu (2006): Aggregate stability and microbial community dynamics under drying-wetting cycles in a silt loam soil. Soil Biology \& Biochemistry 38, 2053-2062.

DESTATIS (2015): Statistisches Bundesamt. www.destatis.de.

FAO (2017): Unlocking the Potential of Soil Organic Carbon, Outcome Document of the Global Symposium on Soil Organic Carbon. Food and Agriculture Organization of the United Nations. Rome, Italy.

Freibauer, A., Rounsevell, M. D. A., Smith, P. und J. Verhagen (2004): Carbon sequestration in the agricultural soils of Europe. Geoderma 122, 1-23.

Hartge, K.H. und R. Horn (2014): Einführung in die Bodenphysik. 4. Aufl., Schweizerbart, Stuttgart, Deutschland, $372 \mathrm{~S}$.

IPCC (2006): Guidelines for national greenhouse gas inventories. Inst. for Global Environ. Strategies, Hayama, Japan. http://www.ipccnggip.iges.or.jp/ public/2006 gl/ pdf/0_Overview/V0_2_Glossary.pdf.

Janetzko, P. und H. Fleige (1995): Böden als Teile von Landschaften: Watten und Marschen Nordwestdeutschlands, Teil Schleswig-Holstein. In: Blume, H.P., FelixHenningsen, Frede, H.-G., Guggenberger, G., Horn, R. und K. Stahr (Hrsg.): Handbuch der Bodenkunde (Loseblattwerk), Ergänzungslieferung. Wiley-VCH, Weinheim, Deutschland, S. 40.

Landesamt für Geologie und Bergwesen Sachsen-Anhalt (LAGB) (2014): Bodenbericht Sachsen-Anhalt 2014, Grundlagen, Parameter, Hintergrundwerte. Mitteilungen zu Geologie und Bergwesen von Sachsen-Anhalt 18, Halle/Saale, Deutschland, 73 S.

Landesamt für Landwirtschaft, Umwelt und ländliche Räume Schleswig-Holstein (LLUR) (Hrsg.) (2016): Bodenübersichtskarte von Schleswig-Holstein. Flintbek, Deutschland.

Landesamt für Umwelt, Landwirtschaft und Geologie (LfULG) (Hrsg.) (2015): Leitfaden zur Humusversorgnung. Informationen für Praxis, Beratung und Schulung. 1. Aufl., Dresden, Deutschland, 64. S. 
Leifeld, J., Bassin, S. und J. Fuhrer (2005): Carbon stocks in Swiss agricultural soils predicted by land-use, soil characteristics, and altitude. Agriculture, Ecosystems \& Environment 105, 255-266.

Liang, C., Schimel, J.P. und J.D. Jastrow (2017): The importance of anabolism in microbial control over soil carbon storage. Nature Microbiology 2, 17105.

Lugato, E., Bampa, F., Panagos, P., Montanarella, L. und A. Jones (2014): Potential carbon sequestration of European arable soils estimated by modelling a comprehensive set of management practices. Global Change Biology 20, 3557-3567.

MELUND (2018): Ministerium für Energiewende, Landwirtschaft, Umwelt, Natur und Digitalisierung: Landwirtschafts- und Umweltatlas. http://www.umweltdaten.landsh.de/atlas/script/index.php.

Mordhorst, A., Peth, S. und R. Horn (2013): Degradation effects on organic carbon and mechanical strength within aggregates from a Stagnic Luvisol depending on tillage intensity. In: Krümmelbein J., Horn, R. und M. Pagliar (Hrsg.): Soil Degradation. Advances in Geoecology 42, Catena, Reiskirchen, Deutschland, S. 104-128.

Neufeldt, H. (2005): Carbon stocks and sequestration potentials of agricultural soils in the federal state of BadenWürttemberg, SW Germany. Journal of Plant Nutrition and Soil Science 168, 202-211.

Poeplau, C., Don, A., Vesterdal, L., Leifeld, J., Van Wesemael, B.A.S., Schumacher, J. und A. Gensior (2011): Temporal dynamics of soil organic carbon after land-use change in the temperate zone-carbon response functions as a model approach. Global Change Biolology $17,2415-2427$.

Rumpel, C. und I. Kögel-Knabner (2011): Deep soil organic matter - a key but poorly understood component of terrestrial C-cycle. Plant Soil 338, 143-158.

Schiefer, J., Lair, G.J. und W.E.H. Blum (2015): Indicators for the definition of land quality as a basis for the sustainable intensification of agricultural production. International Soil and Water Conservation Research 3, 42-49.

Six, J., Conant, R.T., Paul, E.A. und K. Paustian (2002): Stabilization mechanisms of soil organic matter: Implications for C-saturation of soils. Plant and Soil 241, 155-176.
Springob, G., Brinkmann, S., Engel, N., Kirchmann, H. und J. Böttcher (2001): Organic C levels of Ap horizons in North German Pleistocene sands as influenced by climate, texture, and history of land-use. Journal of Plant Nutrition and Soil Science 164, 681-690.

van Groenigen, J.W., van Kessel, C., Hungate, B.A., Oenema, O., Powlson, D.S. und K.J. van Groenigen (2017): Sequestering Soil Organic Carbon: A Nitrogen Dilemma. Environmental Science \& Technology 51, 47384739 .

Waldmann, F. und W. Weinzierl (2014): Organische Kohlenstoffvorräte der Böden Baden-Württembergs in Abhängigkeit von Bodentyp, Bodenart, Klima und Landnutzung. Reihe KLIMOPASS-Berichte, Landesanstalt für Umwelt, Messungen und Naturschutz Baden-Württemberg, Karlsruhe, Deutschland.

Wiechmann, H. (1995): Böden als Teile von Landschaften: Podsole. In: Blume, H.-P., Felix-Henningsen, Frede, H.-G., Guggenberger, G., Horn, R. und K. Stahr (Hrsg.): Handbuch der Bodenkunde (Loseblattwerk), Ergänzungslieferung. Wiley-VCH, Weinheim, Deutschland, S. 40.

Wiesmeier, M., Spörlein, P., Geuß, U., Hangen, E., Haug, S., Reischl, A., Schilling, B., von Lützow, M. und I. Kögel-Knabner (2012): Soil organic carbon stocks in southeast Germany (Bavaria) as affected by land use, soil type and sampling depth. Global Change Biology $18,2233-2245$.

Wiesmeier, M., Schad, P., von Lützow, M., Poeplau, C., Spörlein, P., Geuß, U., Hangen, E. Reischl, A., Schilling, B. und I. Kögel-Knabner (2014): Quantification of functional soil organic carbon pools for major soil units and land uses in southeast Germany (Bavaria). Agriculture, Ecosystems \& Environment 185, 208-220.

Wördehoff, R., Spellmann, H., Evers, J., Aydın, C.T. und J. Nagel (2012): Kohlenstoffstudie Forst und Holz Schleswig-Holstein. Nordwestdeutsche Forstliche Versuchsanstalt, Göttingen, Deutschland.

Zomer, R.J., Bossio, D.A., Sommer, R. und L.V. Verchot (2017): Global Sequestration Potential of Increased Organic Carbon in Cropland Soils. Scientific Reports 7, 15554. 